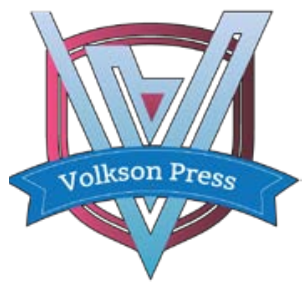

Contents List available at VOLKSON PRESS

New Materials and Intelligent Manufacturing (NMIM) DOI : http://doi.org/10.26480/icnmim.01.2018.161.163

Journal Homepage: https://topicsonchemeng.org.my/

ISBN: 978-1-948012-12-6

\title{
APPLICATION PROPERTIES OF ETHYL BENZOATE: ENVIRONMENTAL- FRIENDLY DYEING CARRIER FOR POLYESTER FIBER
}

\author{
Chang Guan, Yu-Ying Zhou, Qun Liu \\ Jilin Institute of Chemical Technology, Ji Lin \\ *Corresponding Author Email: guanch001@163.com, liuqun198707@163.com
}

This is an open access article distributed under the Creative Commons Attribution License, which permits unrestricted use, distribution, and reproduction in any medium, provided the original work is properly cited

\section{ARTICLE DETAILS}

\section{Article History:}

eceived 26 June 2018 Accepted 2 July 2018 Available online 1 August 2018

\section{ABSTRACT}

This paper uses ethyl benzoate emulsion as dyeing carrier to low temperature dyeing of polyester fiber, inspected the effect of benzyl benzoate on the application properties of polyester fabrics and disperse dyes. Using the thermogravimetric analysis (TGA) method was used to demonstrate that ethyl benzoate as a dyeing carrier had no significant effect on the stability of polyester fibers. Through the effect of the carrier on dye transfer role of dyed fabrics and dyeing rate curves of the Disperse blue 2BLN is investigated. The results show that ethyl benzoate has obvious solubilization and promotion effect on Disperse blue 2BLN.The shade and color fastness of dyed fabric with ethyl benzoate carrier are similar to those with high temperature and high-pressure process and with menthyl salicylate process.

\section{KEYWORDS}

Ethyl benzoate, carrier dying, polyester, application properties

\section{INTRODUCTION}

With the rapid development of science and technology, people have increased the demand for clothing functionality and fashion [1]. Polyester fabrics are strong and durable, strong in recovery, anti-wrinkle and free from scalding. They can be avoided by blending with natural fibers, viscose fibers and wool. Polyester cannot be dyed by traditional high-temperature and high-pressure dyeing $[2,3]$. Carrier dyeing can reduce the dyeing temperature of polyester and avoid Fibers such as protein fibers that are not resistant to high temperatures cause damage [4-7]. However, traditional carriers such as wintergreen oil have heavy odors, large amounts, and certain toxicities, and their applications are limited [8-10]. Therefore, the development and use of dyeing carriers has received increasing attention from researchers [11-13].

Ethyl benzoate is a non-toxic and fruit-flavored chemical raw material. It is mainly used in daily necessities, textile leathers, flavors and fragrances for tobacco and alcohol, food additives, pharmaceuticals, organic synthesis intermediates and solvents, etc. This article uses ethyl benzoate emulsion as a carrier, in line with the requirements of environmentally friendly dyeing carriers. Through the optimization of the dyeing process parameters of the ethyl benzoate carrier, the application performance study, and the comparison of high-temperature and high-pressure dyeing methods and the dyeing effect of methyl salicylate emulsion, the main component of oil of wintergreen, Polyester low temperature dyeing carrier feasibility.

\section{EXPERIMENTAL}

\subsection{Test Materials and Intrument}

Materials:Polyester fabric, 8.3 tex $\times 8.3$ tex, width $147 \mathrm{~cm}$.Disperse blue 2BLN (Zhejiang runtu Co., Ltd.),Acetone (Analytical Pure, Tianjin City DaMao chemical reagents factory), Ethyl benzoate (Analytical Pure, Aladdin Reagents Shanghai Co., Ltd.), Menthyl salicylate (Analytical Pure, Beijing Chemical Factory), Levelling Agent 0-25 (Shandong yousuo Chemical Technology Co., Ltd.), Sodium hydrosulfite (Tianjin City DaMao chemical reagents factory), Saustic soda (Yantai shuangshuang Chemical Co.,Ltd), Neutral soap Tablelets (Shanghai soap factory).

Main Instruments:755B type ultraviolet visible spectrophotometer (Shanghai Jinghua Science and Technology Instrument Co., Ltd.), R-12SMI

type infrared color test machine (Guangzhou Hongjing Experimental Equipment Co., Ltd.), SW-12AII color fastness friction tester (Wenzhou daong textile standard instrument factory), Y (B) 571-II color fastness test machine (Wenzhou daong textile standard instrument factory), YG(B)026H-250 fabric strength instrument (Wenzhou daiong Textile Instrument Co., Ltd.), Datacolor SF650 computer color measurement and matching Color instrument (manufactured by Grandville, Michigan), SDTQ600 synchronous thermal analyzer (TA instruments, USA).

\subsection{Design of dyeing process}

\subsubsection{Technologicai process:}

Dyeing $\rightarrow$ Washing $\rightarrow$ Restore cleaning $\rightarrow$ Washing and drying

\subsubsection{Dyeing process prescription}

(1) Dyeing prescription Polyester fabrics $\quad 1 \mathrm{~g}$ Disperse dyes/\% (o.w.f) $3 \%$ Liquor ratio

Carrier

Levelling Agent 0-25 $\mathrm{pH}$ value

\subsubsection{Dyeing process curve}

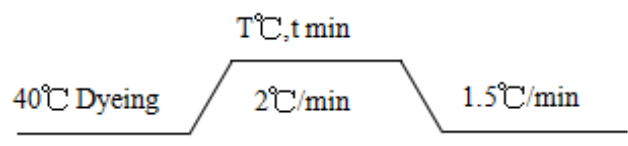

(T-high temperature and high-pressure method is $130^{\circ} \mathrm{C}$, carrier method is $70 \sim 110^{\circ} \mathrm{C}$; $\mathrm{t}$-high temperature and high-pressure method is $40 \mathrm{~min}$, carrier method is $30 \sim 90 \mathrm{~min}$ )

\subsection{Test standard}

\subsubsection{Color testing method}


The K/S value of the dyed fabric was measured at a D65, $10^{\circ}$ light source using a Datacolor SF650 colorimetric colorimeter.

\subsubsection{Breaking strength}

Refer to GB/T 3923.1-2013《Textile fabrics tensile properties - Part 1: Determination of breaking strength and elongation at break method》, using a YG (B) $026 \mathrm{H}-250$ fabric strength tester, tensile strength in warp and weft Measured three times and averaged.

\subsubsection{Staining fastness}

Refer to GB/T 3921-2008 《Color fastness to textiles, soaping color fastness》D (4) method, determine the color fastness to soaping on the $Y$ (B) 571-II wash fastness tester; GB/T 3920-2008 《Textiles color fastness test anti-friction color fastness $》$, measured on the SW-12A II Anti-friction color fastness.

\subsubsection{Polyester fiber thermal stability test}

Take $3 \mathrm{~g} / \mathrm{L}$ of ethyl benzoate carrier and distilled water were used to treat $1 \mathrm{~g}$ of polyester fabric. The liquor ratio was $1: 50$, and the temperature is $100^{\circ} \mathrm{C}$ for $40 \mathrm{~min}$. The sample mass was $5 \mathrm{mg}$. The SDT-Q600 thermogravimetric analyzer is used for testing. Test conditions: air atmosphere, heating rate $10^{\circ} \mathrm{C} / \mathrm{min}$, temperature: room temperature $\sim 500^{\circ} \mathrm{C}$.

\subsubsection{Migration index}

Use Disperse blue 2BLN to dye polyester fabrics. Divide them into several swatches of equal mass and then combine them with undyed fabrics of the same specifications and quality. Suture, refer to section 1.2.2, according to the formula (1) Calculate the migration MI value. The higher MI value, the higher the dyeing rate and the better the migration.

$$
\text { MI value }=\frac{(\mathrm{K} / \mathrm{S})_{1}}{(\mathrm{~K} / \mathrm{S})_{2}} \times 100 \%
$$

In the formula:

(K/S)1-_The color depth of the white fabric after transfection

(K/S)2- - The color depth of primary fabrics after dyeing

\subsubsection{Dye rate}

Using residual liquid colorimetric method, the dyeing stock solution and raffinate were first collected in a $500-\mathrm{mL}$ volumetric flask and brought to volume; then $6 \mathrm{~mL}$ was removed from a $500-\mathrm{mL}$ volumetric flask and placed in a $10-\mathrm{mL}$ volumetric flask, and $4 \mathrm{~mL}$ of acetone was added (When the ratio of water to acetone is $3: 2$, the dye is completely dissolved.) The absorbance is measured at the maximum absorption wavelength, and the dye uptake percentage is calculated according to formula (2).

$$
\mathrm{E} / \%=\left(1-\frac{\mathrm{A}_{1}}{\mathrm{~A}_{0}}\right) \times 100
$$

In the formula: $\mathrm{E}$ is the percentage of dyeing, $\mathrm{A} 1$ is the residual absorbance, and $\mathrm{A} 0$ is the absorbance of the original solution.

\subsubsection{Dyeing rate curve of disperse dyes at constant temperature}

Polyester fabrics were dyed with disperse blue 2BLN, and samples were taken every $10 \mathrm{~min}$ at $100^{\circ} \mathrm{C}$ for $80 \mathrm{~min}$ at room temperature until the end of the dyeing process. Dyeing raffinates were collected and the dye rate was determined according to the 1.3.6 method. The constant temperature dyeing rate curve was plotted.

\section{EXPERIMENTAL RESULTS AND ANALYSIS}

\subsection{Effect of ethyl benzoate carrier on the application performance of polyester fabric}

\subsubsection{Effect of thermal sTableility of ethyl benzoate carrier polyester fabrics}

Samples of fabrics treated with ethyl benzoate and distilled water were subjected to thermogravimetric analysis (TGA) using a SDT-Q600 thermogravimetric analyzer, as shown in Figure 1

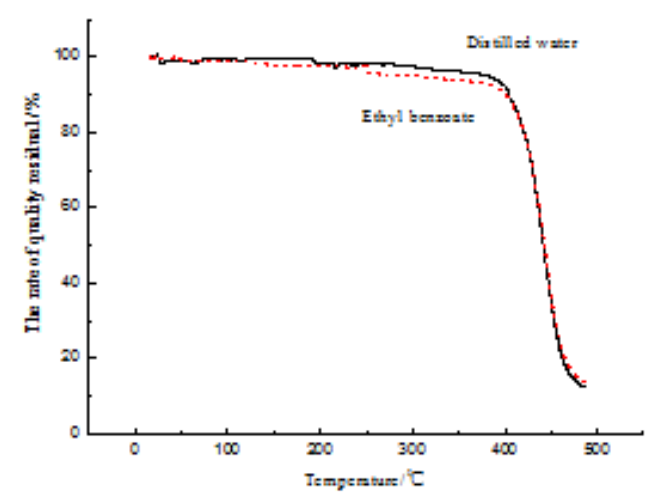

Figure 1: Thermogravimetric curves of polyester fiber

From Figure 1, the weight loss rate of the polyester sample after the carrier treatment is slightly larger than that of the distilled water treatment sample because the carrier attached to the fiber is decomposed. Both the initial decomposition temperature and the termination decomposition temperature are basically the same, and it can be considered that the carrier treatment will not cause the degradation of the fiber macromolecules and will not affect the thermal stability of the polyester fabric.

\subsubsection{Effect of ethyl benzoate on tensile properties of polyester fabrics}

The effects of ethyl benzoate carrier on the tensile properties of polyester fabrics are shown in Table 1.

Table 1: Effect of carrier ethyl benzoate on the tensile properties of polyester fiber

\begin{tabular}{ccccc}
\hline & \multicolumn{2}{c}{ Breaking strength/N } & \multicolumn{2}{c}{ Elongation at break/\% } \\
Carrier & Meridional & zonal & Meridional & zonal \\
& 668.8 & 632.01 & $34.81 \%$ & $28.99 \%$ \\
$\begin{array}{c}\text { Distilled } \\
\text { water } \\
\begin{array}{c}\text { Ethyl } \\
\text { benzoate }\end{array}\end{array}$ & 662.34 & 630.26 & $36.70 \%$ & $33.40 \%$ \\
\hline
\end{tabular}

From Table 1 that the breaking strength of the polyester fabric treated with ethyl benzoate carrier is slightly lower than that of the distilled water treated polyester fabric, and the elongation at break is slightly higher than that of the polyester fabric treated with distilled water.

In summary, the promotion mechanism of the carrier only increases the cavities between the micromolecular chains, does not cause degradation of the fiber macromolecules, and does not cause damage to the polyester fabrics.

\subsection{Effect of ethyl benzoic on dyeing properties of disperse dyes}

\subsubsection{Effect of ethyl benzoate on dye transfer role of dyed fabrics}

The effect of ethyl benzoate on the disperse blue 2BLN dye migration performance is shown in Figure 2.

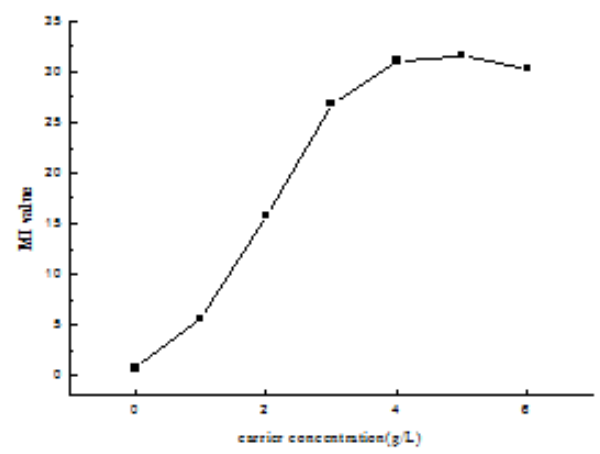

Figure 2: Effect of carrier concentration on MI of dyed fabric 
As can be seen from Figure 2, with the increase of the carrier mass concentration, the migration dye index of the disperse dyes becomes significantly larger; when the carrier mass concentration is higher than 4 $\mathrm{g} / \mathrm{L}$, the disperse dye migration rate increases slowly and decreases. This is because the carrier has an affinity for dyes and fibers, it can carry more dyes from the fiber surface to the fiber internal migration, when the carrier mass concentration increases to a certain extent, the dye solution will dissolve more dye, but make more Dye remains in the dye solution, reducing the dye uptake rate, resulting in decreased dyeing fabric dye transfer rate. Therefore, it was confirmed that ethyl benzoate improves the dyeing performance of polyester dyed fabrics.

\subsubsection{Effect of ethyl benzoate on the dyeing rate of disperse dyes at constant temperature}

The constant temperature dyeing rate curve of ethyl benzoate to disperse blue 2BLN is plotted with time and dye rate, as shown in Figure 3.

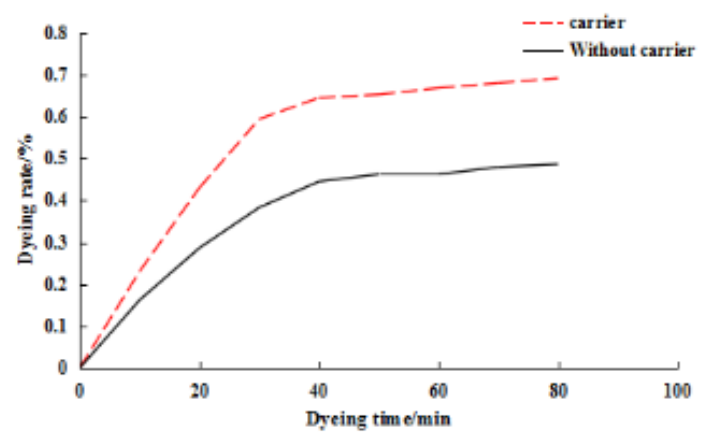

Figure 3: Dye up-taking rate curves at constant temperature

From Figure 3, it can be seen that in the dyeing liquid at the beginning of dyeing, the dyeing rate of the fabric dyed with the ethyl benzoate carrier increases rapidly, and the dyeing balance is prioritized, and the equilibrium dye uptake rate is significantly higher than that without the carrier.In summary, it can be concluded that ethyl benzoate as a carrier can effectively solubilize disperse dyes and promote dyeing during the dyeing process of polyester.

3.3 Comparison of the dyeing effect between ethyl benzoate and other methods

Under the optimal dyeing conditions described above, Levelling Agent 025 was emulsified with methyl salicylate in the optimal mass ratio of 1:10. Comparison of ethyl benzoate dyed fabrics with the main component of menthyl salicylate carrier and the dyeing effect of esters and High temperature and high pressure dyeing fabrics is shown in Table. 3.

Table 3: Three methods dyeing effect of polyester fabric after dyeing

\begin{tabular}{|c|c|c|c|c|c|}
\hline \multirow[t]{2}{*}{ Dyeing carrier } & \multirow{2}{*}{$\begin{array}{c}\mathrm{K} / \mathrm{S} \\
\text { value }\end{array}$} & \multicolumn{2}{|c|}{$\begin{array}{l}\text { Soap wash } \\
\text { fastness } \\
\text { grade/grade }\end{array}$} & \multicolumn{2}{|c|}{$\begin{array}{c}\text { Anti-friction } \\
\text { color } \\
\text { fastness/grade }\end{array}$} \\
\hline & & $\begin{array}{c}\text { Stainin } \\
\mathrm{g}\end{array}$ & fading & Dry & Wet \\
\hline ethyl benzoate & 13.405 & $4-5$ & $4-5$ & 5 & $4-5$ \\
\hline $\begin{array}{l}\text { menthyl } \\
\text { salicylate }\end{array}$ & 14.165 & $4-5$ & 4 & 5 & $4-5$ \\
\hline $\begin{array}{c}\text { High } \\
\text { temperature } \\
\text { and high } \\
\text { pressure } \\
\text { dyeing }\end{array}$ & 14.710 & $4-5$ & 4 & 5 & $4-5$ \\
\hline
\end{tabular}

From Table 3. that the K/S value of the ethyl benzoate dyeing fabric is slightly lower than that of the methyl salicylate dyeing method and the high temperature and high pressure dyeing method, but the difference is not significant. The soaping color fastness (staining and fading) and dry and wet rubbing fastness of the three methods of dyeing fabrics are all good, basically above grade 4 .
Ethyl benzoate can be used as a dyeing mechanism for polyester dyeing at low temperatures. The mechanism only increases the cavities between the micromolecular chains, does not cause the degradation of macromolecules, and does not cause damage to polyester fabrics. Ethyl benzoate has a certain solubilizing and accelerating effect on Disperse blue 2BLN, which can improve the disperse dye's migration property, equilibrium dye rate and dye rate.

The K/S value and the color fastness of the ethyl benzoate dyeing fabric are similar to the high temperature and high pressure dyeing method, the methyl salicylate dyeing fabric, the main component of the wintergreen oil carrier, and ethyl benzoate as the dyeing carrier. And the dyeing temperature is lower and the carrier is used less.

\section{REFERENCES}

[1] Tang, J., Zan-Min, W. 2013. The dyeing of polyester textiles with new environmentally friendly carriers [J]. Dyeing and Finishing, 39 (14), 14-17.

[2] Radei, S.F., Carrión-Fité, J., Ardanuy, M. 2018. Kinetics of Low Temperature Polyester-r Dyeing with High Molecular Weight Disperse Dyes by Solvent Microemulsion and Agro Sourced Auxiliaries [J]. Polymers, 10 (2), 02-11.

[3] Jia, M.L., Jing, M., Kunlun, W. 2014. Application of vanillin in low temperature disperse dyeing of polyester [J]. Dyeing and Finishing, 40 (10), 24-27.

[4] Jing, M., Kunlun, W. 2014. Application of vanillin in low temperature disperse dyeing of polyester [J]. Dyeing and Finishing, 40 (10), 24-27.

[5] Fan, Y.L., Wang, X.Y. 2017. Study on the influence of carrier complexing on the atmospheric boiling dyeing of terylene disperse dyes [J]. Synthetic Fibers, 46 (01), 41-45.

[6] Carrion-Fite, F.J., Radei, S. 2017. Development auxiliaries for dyeing polyester with disperse d-yes at low temperatures [J]. Material science and engineering, 254 (8), 082-006.

[7] Jiang, Y.Y. 2017. Low-temperature dyeing of thymol from polyester [J]. Dyeing and Finishing, 43 (1), 28-30.

[8] Jia, M.L., Wang, C. 2016. Application properties of benzyl benzoate: environmentalriendly dyeing carrier of polyester fiber [J]. Silk, 53 (4), 1317.

[9] Jia, M.L. 2015. Low temperature dyeing of cinnamic acid carrier in polyester fabrics [J]. Dyeing and Finishing, 41 (4), 16-20.

[10] Zhu, H.Y., Cao, P., Jia, M.L. 2017. Carrier Dyeing of Polyester/Spandex Knitted Fabric with Benzyl Benzoate [J]. Progress in Textile Science \& Technology, (1), 35-39.

[11] Jia, M.L. 2016. Polyester benzoyl benzoate dyeing [J]. Dyeing and Finishing, 42(04), 22-25.

[12] Song, H.J., Gao, Y.M.Z.W. 2011. Research on the application of environmental protection and tasteless carrier CWP810 in polyester dyeing [J]. Silk, 48 (7), 11-14.

[13] Min, K.J. 1982. Application of Expanding Agent OP and Methyl Salicylate in the Dyeing of Polyester and Blended Fabrics [J]. Dyestuff Industry, (05), 40-42+36.

\section{CONCLUSION}

